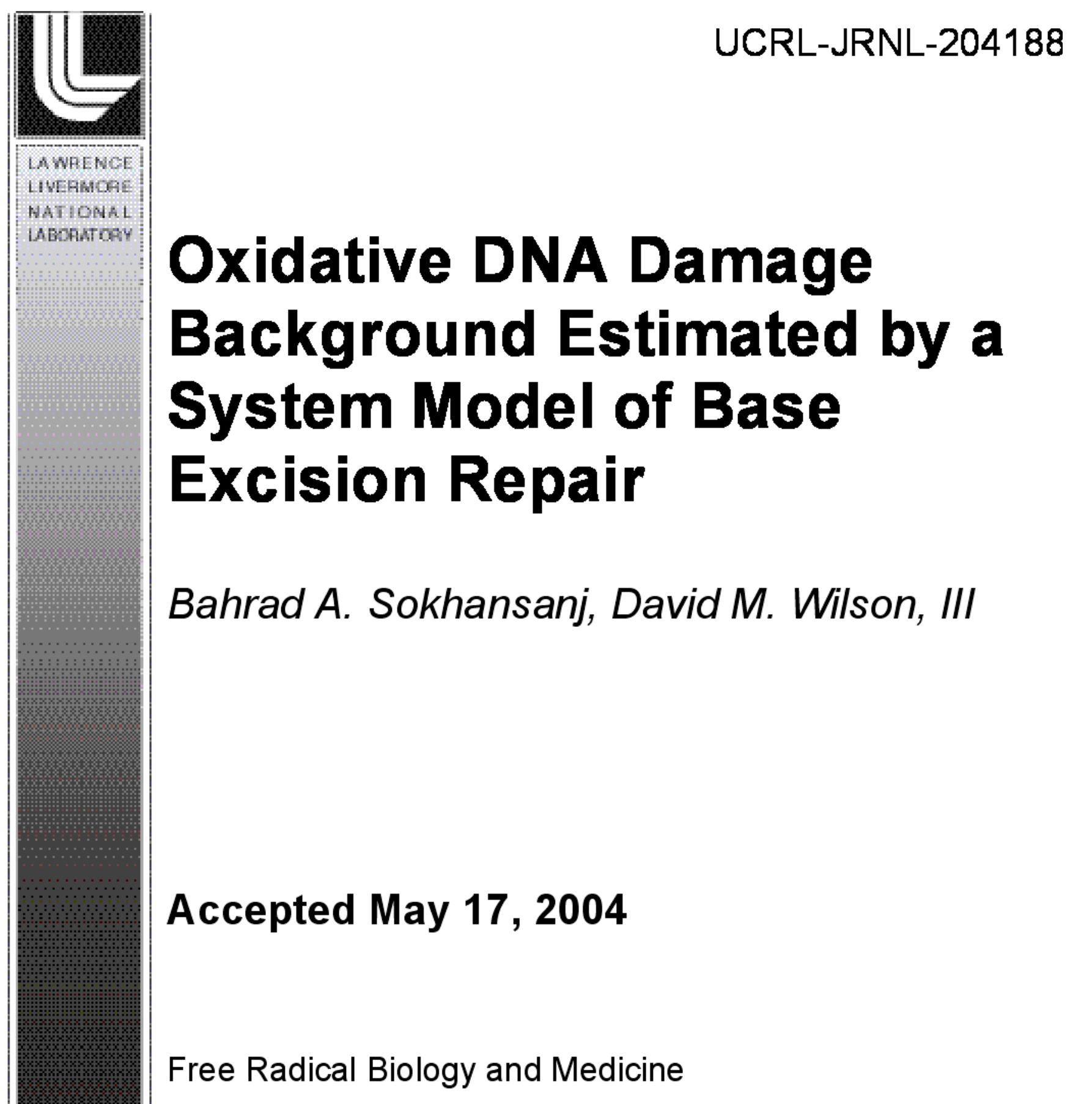


This document was prepared as an account of work sponsored by an agency of the United States Government. Neither the United States Government nor the University of California nor any of their employees, makes any warranty, express or implied, or assumes any legal liability or responsibility for the accuracy, completeness, or usefulness of any information, apparatus, product, or process disclosed, or represents that its use would not infringe privately owned rights. Reference herein to any specific commercial product, process, or service by trade name, trademark, manufacturer, or otherwise, does not necessarily constitute or imply its endorsement, recommendation, or favoring by the United States Government or the University of California. The views and opinions of authors expressed herein do not necessarily state or reflect those of the United States Government or the University of California, and shall not be used for advertising or product endorsement purposes.

This work was performed under the auspices of the U.S. Department of Energy by University of California, Lawrence Livermore National Laboratory under Contract W-7405-Eng-48. 


\title{
Oxidative DNA damage background estimated by a system model of base excision repair
}

\author{
Bahrad A. Sokhansanj ${ }^{1, *}$, David M. Wilson III $^{2}$ \\ ${ }^{1}$ Chemistry \& Materials Science Directorate, L-235, University of California, Lawrence \\ Livermore National Laboratory, 7000 East Avenue, Livermore, CA 94551-9900, \\ ${ }^{2}$ Laboratory of Molecular Gerontology, National Institute on Aging, 5600 Nathan Shock \\ Drive, Baltimore, MD 21224-6825.
}

*To whom correspondence should be addressed: Phone (925) 422-8643, Fax (925) 422-

3570, Email: sokhansanj@1lnl.gov.

Acknowledgements. We thank our colleagues at Livermore, Drs. J. P. Fitch, G. H. Rodrigue, A. A. Quong, I.

M. Jones, and H. W. Mohrenweiser, as well as Dr. N. C. de Souza-Pinto at NIA, for their support and input.

BAS was supported during some of this research by a LLNL Student Employee Graduate Research

Fellowship. DMW was supported in part by the Biological and Environmental Research Program, U.S.

Department of Energy, Interagency Agreement No. DE-AI02-02ER63424. This work was performed under

the auspices of the U.S. DOE by the University of California, LLNL under Contract No. W-7405-Eng-48.

RUNNING TITLE: Oxidative DNA damage model

MANUSCRIPT NUMBER: FRBM HP 111586 


\title{
Oxidative DNA damage background estimated by a system model of base excision repair
}

\begin{abstract}
Human DNA can be damaged by natural metabolism through free radical production. It has been suggested that the equilibrium between innate damage and cellular DNA repair results in an oxidative DNA damage background that potentially contributes to disease and aging. Efforts to quantitatively characterize the human oxidative DNA damage background level based on measuring 8-oxoguanine lesions as a biomarker have led to estimates varying over 3-4 orders of magnitude, depending on the method of measurement. We applied a previously developed and validated quantitative pathway model of human DNA base excision repair, integrating experimentally determined endogenous damage rates and model parameters from multiple sources. Our estimates of at most 100 8-oxoguanine lesions per cell are consistent with the low end of data from biochemical and cell biology experiments, a result robust to model limitations and parameter variation. Our results show the power of quantitative system modeling to interpret composite experimental data and make biologically and physiologically relevant predictions for complex human DNA repair pathway mechanisms and capacity.
\end{abstract}

\section{KEYWORDS}

oxidative DNA damage, base excision repair, pathway modeling, simulation 


\section{INTRODUCTION}

DNA is continuously damaged by endogenous sources, namely reactive oxygen species (ROS) formed during normal cellular respiration. The resulting background damage can contribute to aging and disease development, and thus must be better defined to accurately assess health risks associated with environmental exposures. Among the many different kinds of damaged bases that can ensue, a common representative biomarker for oxidative DNA damage is the number of 8-oxoguanine (8-oxoG) lesions per genome. Numerous quantitative experimental measurements of normal steady-state 8-oxoG levels have been made over the past decade, resulting in estimates that vary from $10^{2}$ to $10^{5}$ lesions per cell $[1,2]$. To approximate the steady state number of lesions based on knowledge of endogenous formation rates and repair mechanisms, we apply a previously developed and validated quantitative model [3] of base excision repair (BER), the major pathway responsible for correcting spontaneous, alkylation, and oxidative DNA damage in humans [4]. Simulations incorporating physiological rates of DNA damage formation and repair robustly predict that the steady state levels of 8-oxoG, spontaneously formed abasic sites, and BER damage intermediate products total $\sim 100$ lesions per cell, arguing for background levels at the low end of experimental estimates.

\section{MODELING AND RESULTS}

BER (the current model is presented in Figure 1) is a complex system of coordinated sequential enzymatic reactions. Briefly, for the case of oxidative damage, e.g. 8-oxoG, the pathway is initiated by a DNA glycosylase [i.e. 8-oxoG DNA glycosylase (Ogg1)], which recognizes and removes damaged bases through hydrolysis of the N-glycosylic bond [5]. Some DNA glycosylases (e.g. Ogg1) also exhibit an AP lyase activity, incising the DNA backbone 3' to the AP residue, leading to a sub-pathway (Pathway A) in which AP 
endonuclease (Ape1) removes the 3'-obstructive end [6]. However, in most cases of AP site repair, the DNA is incised 5' to the lesion by Ape1 [7], generating a 5'-deoxyribose phosphate (dRp), which can be removed by DNA Polymerase $\square$ (Pol $\square$ ) [8]. Short-patch repair synthesis (Pathway B) consists of single base replacement by DNA Pol $\square$, followed by ligation by DNA Ligase 1 (Lig1) or an Xrcc1/Lig3 complex [8, 9, 10]. Long-patch repair synthesis (Pathway C) involves gap-filling of 2-7 nucleotides by Pol $\square[11]$ or by Pol $\square \square$ in cooperation with PCNA [12], followed by excision of the flap-like DNA structure by flap endonuclease 1 (Fen1), with sealing of the final nick by Lig1 or Xrcc1/Lig3.

Our comprehensive BER model (Figure 1) is based on the current biochemical and biological understanding of the pathway, and its reactions and parameters are designed to best represent a typical cellular environment. Our model applies Michaelis-Menten kinetics and the assumptions detailed in Sokhansanj, et al. 2002 [3]. We have since modified the original model to account for information learned after the initial publication, primarily recent data on the kinetic role of Lig3 [13], allowing us to fully include its activity as a reaction step in the comprehensive BER model. Details of the changes are described in Supplementary Material, along with equations, kinetic parameters, and protein concentrations (Supplementary Table S1) used to define the comprehensive model.

We simulated the comprehensive BER model to estimate steady state amounts of DNA lesions and BER pathway intermediates for in vivo levels of 8-oxoG $\left(f_{0}\right)$ and AP sites $\left(f_{1}\right)$. We focused on these lesions since (i) abasic sites and 8-oxoG are major mutagenic damages, (ii) there are multiple published measurements of Ape1 and Ogg1 activities [3], and (iii) there has been substantial effort by different laboratories using a variety of experimental methods to obtain accurate measurements of 8-oxoG in vivo as a biomarker for oxidative DNA damage in general (e.g. those reviewed in [2]). An estimated 100-500 8- 
oxoG sites are formed per cell per day [14]. While urine testing estimates the creation of 10,000 8-oxoG/cell/day from metabolic ROS [15], this number likely includes damage created during sample processing and analysis. Extrapolating from DNA exposed to high temperatures, it has been estimated that $\sim 2,000-10,000$ apurinic and 20-100 apyrimidinic sites are formed spontaneously per mammalian genome per day [14]. Using a different method, a similar rate of $\sim 9,000 \mathrm{AP}$ sites/cell/day was determined [16].

Our model implements constant formation rates of 500 8-oxoG/day and 10,000 AP/day. Using these rates, the model predicts $\sim 53$ total lesions/cell: approximately one 8oxoG site and 12 abasic sites, with the remaining being repair pathway intermediates (mostly due to the slow Pol $\square$-catalyzed dRp lyase step). For 2,000 8-oxoG/day, which would reflect conditions of oxidative stress, such as those attained after intense exercise [17], steady state damage levels increase to 64 lesions/cell (6 8-oxoG; 13 AP sites). For 10,000 8-oxoG/day (and 10,000 AP sites/day), the model predicts 127 total lesions (28 8oxoG; 22 AP sites). Steady state numbers of individual repair intermediates are provided in Supplementary Table S2.

Figure 2A shows a linear increase in steady state lesion numbers with increasing 8oxoG formation rates. At about 125,000 8-oxoG/cell/day, there is no steady state solution to the equations and damage accumulates uncontrollably (representing the predicted maximum damage capacity). Just before this "breaking point", the maximum steady state 8oxoG damage is about 900 total lesions/cell. The breaking point for AP site repair is well above $10^{6} \mathrm{AP} /$ cell/day (results not shown).

We investigated the dependency of our prediction on repair protein concentration to account for potential inaccuracy in experimental estimates and biologically relevant cell 
type variation, particularly for highly abundant Pol $\square$, Ogg1, and Ape1 (50-300,000/cell). As Figure 2B shows, a noticeable impact on predicted lesions/cell is observed for 25,000 or fewer repair proteins/cell, but predicted levels are always on the order of $10^{2}$. (Results are identical for 5,000 8-oxoG/cell/day; not shown). In estimating protein concentration we assumed that Pol $\square$, Ogg1, and Ape1 are uniformly distributed throughout the whole cell. If they are more prevalent in the nucleus, concentrations will be higher than those used in the model resulting in lower steady state damage levels.

We studied the sensitivity to reaction rate by adjusting the $\mathrm{K}_{\mathrm{M}}$ of all reactions. Notably, to better reflect physiological conditions within the nucleus leading to a reduced diffusion constant, we already used a 10 -fold higher $\mathrm{K}_{\mathrm{M}}$ than measured for all enzymes in the simulation (further detailed in Supplementary Material). Above this point, the predicted number of steady state lesions per cell increases linearly with $\mathrm{K}_{\mathrm{M}}$ (Figure $2 \mathrm{C}$ ).

Another potential cause for higher levels of steady state damage is incomplete repair following a transient "spike" of damage. Figure 2D shows that, assuming a background of 500 8-oxoG/day and 10,000 AP/day, an 8-oxoG damage "spike" of up to 20,000 8-oxoG sites/cell is essentially completely repaired within less than an hour, with transient numbers of repair intermediates increasing to a peak of up to 4,600 before dropping back to normal steady state levels. A spike of AP sites would be repaired even more quickly than 8-oxoG lesions, and results are almost identical given 5,000 8-oxoG/day (data not shown). Notably, all of our simulations assume a substantial increase in the 8-oxoG DNA glycosylase reaction kinetics of Ogg1 in the presence of Ape1 (possibly due either to pathway coordination or enzyme cooperativity) [3]. Simulations with 10-fold reduced Ogg1 kinetics result in a corresponding 10 -fold increase in 8 -oxoG lesions and no other repair intermediate species; consequently, the total steady state number of lesions is only slightly 
elevated (e.g., for a 8-oxoG formation rate of 500/cell/day, steady state damage of 65 versus 53 total lesions/cell).

Our estimate that the total number of unrepaired lesions/intermediates resulting from 8-oxoG and AP site damage is at most $\sim 100$ sites per cell contrasts several experimental steady state measurements of as many as 400,000 8-oxoG residues and 200,000 AP sites per genome $[1,18]$. This high level of measured damage, which often includes repair processing intermediates, has been argued to be at least in part the result of hydrolysis and/or oxidation during the isolation and handling of DNA [1, 19]. The most recent experimental measurements $[2,20]$ have shown further reduction in estimates, with the lowest direct measurements using chromatography yielding about 10,000 lesions/cell. In addition, our prediction that steady-state damage levels are linearly proportional to the damage formation rate at magnitudes comparable to those here agree qualitatively with the dependence on radiation dose found in [21].

Other estimates based on enzymatic treatment of DNA to convert damaged bases to abasic sites with subsequent quantification by comet assay or chromatography have been as low as 100-800 lesions/cell, near the sensitivity limit of the assay employed [21-24]. Notably, the estimates of the latter approach agree qualitatively with the results of our modeling experiments, indicating that assessments of oxidative DNA base modification employing enzymatic treatment of DNA do not systematically underestimate damage due to incomplete enzyme activity or clustering of modified base sites, for example. Indeed, the glycosylase commonly used to treat DNA in these assays is E. coli Fpg, which also acts to repair oxidatively damaged bases such as FapyAde and FapyGua [19], thus they may even overestimate the amount of 8-oxoG, while being representative of oxidative DNA damage as a whole. In addition, damage measurements based on single cell assays may be more 
representative of the normal states of cells. When cells are pooled, some fraction may be dead or severely damaged: for example, if 5\% have 200,000 lesions/cell and 95\% have 100 lesions/cell, then the estimate will be approximately 10,000 lesions/cell.

Consequently, our "low" damage projections seem reasonable given the potential for mutagenesis and cytotoxicity from even a small number of DNA lesions and the apparent abundance of DNA repair proteins. They are also in agreement with a measurement of about 100 8-oxoG lesions/cell in mouse fibroblasts, which was made by comparing Ogg1knockout mice compared to control - an approach that should be independent of DNA isolation artifacts [25]. Still, there are several issues that warrant further discussion. First, our model excludes product inhibition effects (e.g. for Ogg1 [26]), though product binding is thought to enhance BER coordination, and in turn pathway efficiency, through a "passing the baton" mechanism [27]. Our model also excludes several potential cooperative mechanisms (e.g. the role of Xrcc1) and other compensatory DNA repair pathways. Thus, we believe that the current modeling limitations would balance one another out and have little impact on the final quantitative estimates for steady state DNA damage levels.

Second, our model excludes other mechanisms of base modification that may give rise to BER-type intermediates. However, experimental measurements suggest that other base lesions (e.g. 8-oxoadenine, uracil, 3-methyladenine, thymine glycol, etc. and abasic sites resulting from their excision) would (i) occur at about the same rate as 8-oxoG or less [14], (ii) be repaired faster by DNA glycosylases [3], and (iii) contribute perhaps no more than a comparable amount of damage dependent on the experimental measurement method $[22,28]$. While we have neglected the role of Ogg1 in repairing other lesions besides 8oxoG (such as 8-oxoadenine [19]), the cellular abundance of Ogg1 (10 5 proteins/cell) is sufficient to catalyze orders of magnitude more reactions without interfering with its 
specific 8-oxoG glycosylase activity (results not shown). For these reasons along with the linear dependence on formation rate shown in Fig. 2A, alternative forms of base damage will generally have no qualitative impact on our results: given that the low end of experimental estimates of 8-oxoG are supported by our modeling studies, we can reasonably expect a proportionately low steady state number of other oxidative lesion levels.

Third, our model excludes mitochondria, a major site for ROS production and potential DNA damage. However, (i) there is perhaps 100-fold less mtDNA than nuclear DNA, (ii) studies indicate that oxidized base frequencies are only 2-3 times greater [29], and (iii) Ogg1 repair may be 1.7 times faster in the mitochondria [30].

Finally, the $\sim 2-8$ fold difference between our modeling predictions and the low experimental measurements may arise due to chromatin structure effects, where studies have shown that nucleosomes and chromatin can inhibit BER, specifically DNA Pol $\square$, DNA ligase [31,32] and Ape1 [33] up to about 10-fold. Efforts are now underway to incorporate the inhibitory impact of chromatin on BER into our model, and examine its effect on repair capacity and DNA damage buildup.

\section{CONCLUSIONS}

The system-level modeling efforts described within demonstrate the utility of complex quantitative models to address biological issues relating to critical cellular function and pathways. Using 8-oxoG as an oxidative damage biomarker, our system model of the human BER pathway reveals that when incorporating experimentally determined rates of endogenous oxidative DNA damage formation and BER kinetics with knowledge of repair 
mechanisms, steady-state background oxidative damage levels in humans are consistent with both cell biology data and the lowest range of experimental estimates made using enzymatic treatment of modified DNA bases - a result that holds generally firm in the face of variation in DNA damage formation rate, repair protein concentration, and kinetics. 


\section{REFERENCES}

[1] Dizdaroglu, M.; Jaruga, P.; Birincioglu, M.; Rodriguez, H. Free radical-induced damage to DNA: mechanisms and measurement. Free Radic. Biol. Med. 32: 1102-115; 2002.

[2] European Standards Committee on Oxidative DNA Damage (ESCODD). Measurement of DNA oxidation in human cells by chromatographic and enzymic methods. Free Radic.

Biol. Med. 34: 1089-1099; 2003.

[3] Sokhansanj, B. A.; Rodrigue, G. R.; Fitch, J. P.; Wilson, D. M., 3rd. A quantitative model of human DNA base excision repair: I. Mechanistic insights. Nucleic Acids Res. 30: $1817-1825 ; 2002$.

[4] Wilson, D. M., 3rd; Sofinowski, T. M.; McNeill, D. R. Repair mechanisms for oxidative damage. Front Biosci. 8: d963-d981; 2003.

[5] Scharer, O. D.; J. Jiricny, J. Recent progress in the biology, chemistry and structural biology of DNA glycosylases. Bioessays. 23: 270-281; 2001.

[6] Pascucci, B.; Maga, G.; Hubscher, U.; Bjoras, M.; Seeberg, E.; Hickson, I. D.; Villani, G.; Giordano, C.,;Cellai, L.; Dogliotti, E. Reconstitution of the base excision repair pathway for 7,8-dihydro-8-oxoguanine with purified human proteins. Nucleic Acids Res. 30: 2124-2130; 2002.

[7] Wilson, D. M. 3rd; Barsky, D. The major human abasic endonuclease Ape1: formation, consequences and repair of abasic lsions in DNA. Mutat. Res. 484: 283-307; 2001.

[8] Wilson, S. H. Mammalian base excision repair and DNA polymerase beta. Mutat. Res. 407: 203-215; 1998.

[9] Tomkinson, A. E.; Mackey, Z. B. Structure and function of mammalian DNA ligases. Mutat. Res. 407: 1-9; 1998. 
[10] Caldecott, K. W. XRCC1 and DNA strand break repair. DNA Repair (Amst.). 2: 955$969 ; 2003$.

[11] Podlutsky, A. J.; Dianova, I. I.; Podust, V. N.; Bohr, V. A.; Dianov, G. L. Human DNA polymerase beta initiates DNA synthesis during long-patch repair of reduced AP sites in DNA. EMBO J.; 20: 1447-1482; 2001.

[12] Matsumoto, Y.; Kim, K.; Hurwitz, J.; Gary, R.; Levin, D. S.; Tomkinson, A. E.; Park, M. S. Reconstitution of proliferating cell nuclear antigen-independent repair of apurinic/apyrimidinic sites with purified human proteins. J. Biol. Chem. 274: 33703-33708; 1999.

[13] Ho, E. L. Y.; Satho, M. S. Repair of single-strand DNA interruptions by redundant pathways and its implication in cellular sensitivity to DNA-damaging agents. Nucleic Acids Res. 31: 7032-7040; 2003.

[14] Lindahl, T. Instability and decay of the primary structure of DNA. Nature. 362: 709$715 ; 1993$.

[15] Richter, C.; Park, J. W.; Ames, B. N. Normal oxidative damage to mitochondrial and nuclear DNA is extensive. Proc. Natl. Acad. Sci.USA. 85: 6465-6467; 1988.

[16] Nakamura, J.; Walker, V. E.; Upton, P. B.; Chiang, S. Y.; Kow, Y. W.; Swenberg, J. A. Highly sensitive apurinic/apyrimidinic site assay can detect spontaneous and chemically induced depurination under physical conditions. Cancer Res. 58: 222-225; 1998.

[17] Tsai, K.; Hsu, T. G.; Hsu, K. M.; Cheng, H.; Liu, T. Y.,;Hsu, C. F.; Kong, C. W. Oxidative DNA damage in human peripheral leukocytes induced by massive aerobic exercise. Free Radic. Biol. Med. 31: 1465-1472; 2001. 
[18] Atamna, H.; Cheung, I.; Ames, B. N. A method for detecting abasic sites in living cells: age-dependent change in base excision repair. Proc. Natl. Acad. Sci. USA. 97: 686$691 ; 2000$.

[19] Cadet, J.; Bellon, S.; Berger, M.; Bourdat, A. G.; Douki, T.; Duarte, V.; Frelon, S.;

Gasparutto, D.; Muller, E.; Ravanat, J. L.; Sauviago, S. Recent aspects of oxidative DNA damage: guanine lesions, measurement and substrate specificity of DNA repair glycosylases. Biol. Chem. 383: 933-943; 2002.

[20] European Standards Committee on Oxidative DNA Damage (ESCODD). Comparative analysis of baseline 8-oxo-7,8-dihydroguanine in mammalian cell DNA, by different methods in different laboratories: an approach to consensus. Carcinogenesis. 23: 2129$2133 ; 2002$.

[21] Pouget, J. P.; Douki, T.; Richard, M. J.; Cadet, J. DNA damage induced in cells by gamma and UVA radiation as measured by HPLC/GC-MS and HPLC-EC and Comet assay. Chem. Res. Toxicol. 13: 541-549; 2000.

[22] Pflaum, M.; Will, O.; Epe, B. Determination of steady-state levels of oxidative DNA base modifications in mammalian cells by means of repair endonucleases. Carcinogenesis, 8: 2225-2231; 1997.

[23] Collins, A. R.; Horvathova, E. Oxidative DNA damage, antioxidants and DNA repair: applications of the comet assay. Biochem. Soc. Trans. 29: 337-341; 2001.

[24] Jaruga, P.; Speina, E.; Gackowski, D.; Tudek, B.; Olinski, R. Endogenous oxidative DNA base modifications analysed with repair enzymes and GC/MS technique. Nucleic Acids Res. 28: e16; 2000. 
[25] Klungland, A.; Rosewell, I.; Hollenbach, S.; Larsen, E.; Daly, G.; Epe, B.; Seeberg, E.; Lindahl, T.; Barnes, D. E. Accumulation of premutagenic DNA lesions in mice defective in removal of oxidative base damage. Proc. Natl. Acad. Sci. USA, 96: 1330013306, 1999.

[26] Vidal, A. E.; Hickson, I. D.; Boiteux, S.; Radicella, J. P. Mechanism of stimulation of the DNA glycosylase activity of hOGG1 by the major human AP endonuclease: bypass of the AP lyase activity step. Nucleic Acids Res. 29: 1285-1292; 2001.

[27] Wilson, S. H.; Kunkel, T. A. Passing the baton in base excision repair. Nature Struct, Biol. 7: 176-178; 2000.

[28] Podmore, I. D.; Griffiths, H. R.; Herbert, K. E.; Mistry, N.; Mistry, P.; Lunec, J. Vitamin C exhibits pro-oxidant properties. Nature, 392: 559, 1998.

[29] Hudson, E. K. ; Hogue, B. ; Souza-Pinto, N. C. de; Croteau, D. L.; Anson, R. M.;

Bohr, V. A.; Hansford, R. G. Age-associated change in mitochondrial DNA damage. Free Radic. Res. 29: 573-579; 1998.

[30] Bohr, V. A.; Stevnsner, T.; Souza-Pinto, N. C. de. Mitochondrial DNA repair of oxidative damage in mammalian cells. Gene. 286: 127-134; 2002).

[31] Li, S.; Smerdon, M. J. Nucleosome structure and repair of N-methylpurines in the GAL1-10 genes of Saccharomyces cerevisiae. J. Biol. Chem. 277: 44651-44659; 2002. [32] Nilsen, H.; Lindahl, T.; Verreault, A. DNA base excision repair of uracil residues in reconstituted nucleosome core particles. EMBO J. 21: 5943-5952; 2002.

[33] Beard, B. C.; Wilson, S. H.; Smerdon, M. J. Suppressed catalytic activity of base excision repair enzymes on rotationally positioned uracil in nucleosomes. Proc. Natl. Acad. Sci. USA. 100: 7465-7470; 2003. 
Figure 1. Schematic of the previously developed BER model [Sokhansanj 2002] with additions as detailed in the text and Supplementary Material. The model includes coordination between Polb dRp lyase and gap filling functions. Multiprotein complexes are not explicitly shown in this schematic, but rate enhancements are given in Supplementary Table S1.

Figure 2. (A) Dependence of abasic site and total lesion (8-oxoG + abasic + intermediates) numbers on 8-oxoG formation rate. (B) Sensitivity of total lesion number to cellular BER enzyme amounts (from top to bottom: Pol $\square$ (black), Ogg1 (grey), Ape1 (black)) for 500 8-oxoG and 10,000 AP sites/cell/day. (C) Effect of multiplying the $\mathrm{K}_{\mathrm{M}}$ of all enzyme activities on abasic site and total lesion levels for 500 (black) and 5,000 (gray) 8-oxoG sites/cell/day. (D) Temporal evolution of the total number of repair intermediates following sudden 8-oxoG lesion production (plotted sequentially as shown), assuming continuous formation of 500 8-oxoG and 10,000 AP sites/day. 


\section{Figure 1.}

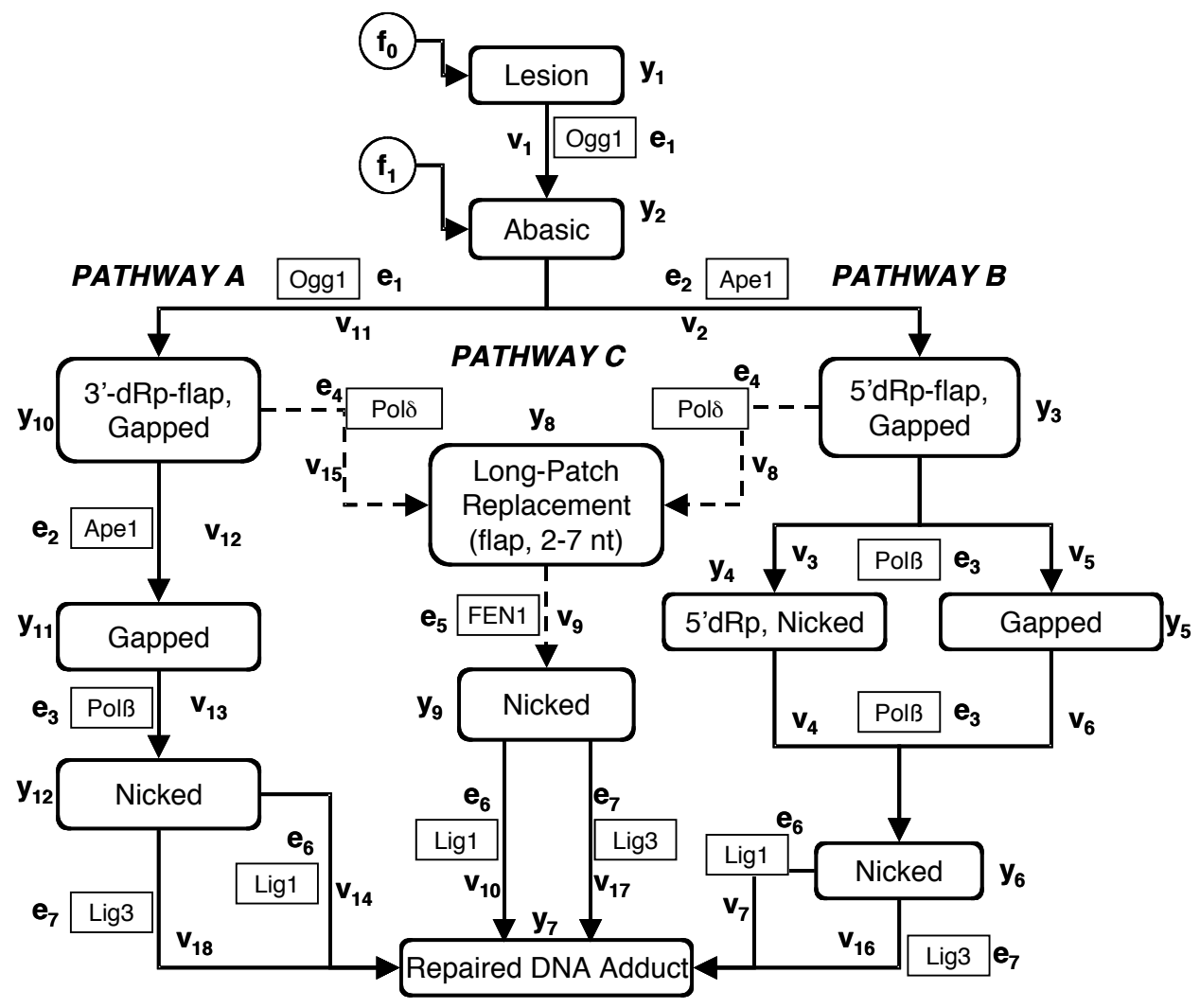

\section{Figure 2}



\section{Oxidative DNA damage background estimated by a system model of base excision repair - SUPPLEMENTARY MATERIAL}

Bahrad A. Sokhansanj, David M. Wilson III

\section{A. Model Details}

The original model [Sokhansanj 2002] (full citations provided below) is revised to include two new cooperative rate enhancements in pathways $\mathrm{A}$ and $\mathrm{C}$. One is a 1.5-fold enhancement in Ogg1 AP lyase activity, provided by Ape1 at approximately twice the concentration of Ogg1 [Saitoh 2000]. The second is a 2-fold increase in Fen1 flapendonuclease cleavage rate when Apel is present at physiological concentrations [Dianova 2001]. Table S1 lists all the kinetic parameters of the model.

The model assumes protein concentrations (listed in Table S1) based on experimental measurements of molecules per cell, with the recognition that these numbers can vary with cell type and condition. In addition, there is evidence, particularly for Ape1 [Evans 2000] and DNA glycosylases such as Ogg1 [Nakabeppu 2001], that repair proteins are distributed within the nucleus and throughout the cell, depending on tissue type. Thus, unless otherwise indicated, we assume BER enzymes are homogeneously distributed, and use a spherical cell with a diameter of $20 \mu \mathrm{m}$ to calculate the molar cellular concentration (Table S1). To compute the concentration of nuclear DNA damage, we presume a spherical nucleus of diameter $5 \mu \mathrm{m}$.

Since the publication of [Sokhansanj 2002], the authors have learned that the physiological level of Lig1 may be 2,000-10,000 molecules per cell [Tomkinson 1990] and (Tomkinson, personal communication), rather than the 50,000 per cell assumed in the previous model. Thus, for these simulations, we assume a number of 5,000 molecules per cell. At that level, DNA ligation by Lig1 becomes the dominant step (i.e. "rate limiting") of BER (data not shown). Also, since the initial publication, we found a recent study of the repair activity of Lig1 and Lig3 on plasmid DNA using radiolabelling in cell extracts and protein overexpression [Ho 2003]. Results were conflicting on the relative role of Lig1 and Lig3 (for some kinds of damage, Lig3 appeared to be responsible for the majority of repair, for others it was evenly divided). Thus, on a preliminary basis we have assumed that Lig1 
and Lig3 have the same kinetics and cellular protein concentration, and that they represent parallel branches for the ligation step in the BER pathway (as shown in Figure 1 of the paper).

Finally, in this work we have multiplied all $K_{M}$ parameters by a factor of 10 to reflect reduced diffusion constants within the nucleus. While the assumption of well-mixing in the nucleus based on single-molecule tracking with fluorescent microscopy of [Sokhansanj 2002] is still assumed to hold, those studies did show reduction in diffusion constant [Phair 2000]. We have elected to reflect that detail as an increase in $K_{M}$ for all BER reactions to remain as physiologically representative as possible. The effect of varying $\mathrm{K}_{\mathrm{M}}$ has been studied by sensitivity analysis (Figure $2 \mathrm{C}$ of the paper). 


\section{B. Model Equations}

The equations describing the BER model (shown in Figure 1 of the paper) are

$$
\begin{aligned}
& \frac{d y_{1}}{d t}=f_{0}(t) \square v_{1} \\
& \frac{d y_{2}}{d t}=f_{1}(t)+v_{1} \square v_{2} \square v_{11} \\
& \frac{d y_{3}}{d t}=v_{2} \square v_{3} \square v_{5} \square v_{8} \\
& \frac{d y_{4}}{d t}=v_{3} \square v_{4} \\
& \frac{d y_{5}}{d t}=v_{5} \square v_{6} \\
& \frac{d y_{6}}{d t}=v_{4}+v_{6} \square v_{7} \\
& \frac{d y_{7}}{d t}=v_{7}+v_{10}+v_{14} \\
& \frac{d y_{8}}{d t}=v_{8}+v_{15} \square v_{9} \\
& \frac{d y_{9}}{d t}=v_{9} \square v_{10} \\
& \frac{d y_{10}}{d t}=v_{11} \square v_{12} \square v_{15} \\
& \frac{d y_{11}}{d t}=v_{12} \square v_{13} \\
& \frac{d y_{12}}{d t}=v_{13} \square v_{14}
\end{aligned}
$$

$$
\begin{aligned}
& v_{1}=y_{1} \frac{k_{1} e_{1}}{y_{1}+K_{1}} \\
& v_{2}=y_{2} \frac{k_{2} e_{2}}{y_{2}+K_{2}} \\
& v_{3}=y_{3} \frac{k_{3} e_{3}}{y_{3}+K_{3}} \\
& v_{4}=k_{4} y_{4} \\
& v_{5}=y_{3} \frac{k_{4} e_{3}}{y_{3}+K_{4}} \\
& v_{6}=k_{3} y_{6} \\
& v_{7}=y_{5} \frac{k_{5} e_{6}}{y_{5}+K_{5}} \\
& v_{8}=y_{3} \frac{k_{6} e_{4}}{y_{3}+K_{6}} \\
& v_{9}=y_{8} \frac{k_{7} e_{5}}{y_{8}+K_{7}} \\
& v_{10}=y_{9} \frac{k_{5} e_{6}}{y_{9}+K_{5}} \\
& v_{11}=k_{8} y_{2} \\
& v_{12}=y_{10} \frac{k_{9} e_{2}}{y_{10}+K_{9}} \\
& v_{13}=y_{11} \frac{k_{3} e_{3}}{y_{11}+K_{3}} \\
& v_{14}=y_{12} \frac{k_{5} e_{6}}{y_{12}+K_{5}}
\end{aligned}
$$

where the parameters $v_{i}$ are defined in Figure 1 , and $k_{i}$ and $K_{i}$, which correspond to $\mathrm{k}_{\mathrm{cat}}$ and $\mathrm{K}_{\mathrm{M}}$ of enzyme reactions respectively, are defined along with $e_{i}$ in Table S1 (Supplementary Material). These equations were derived using Michaelis-Menten kinetics, implying assumptions detailed in our previous work [Sokhansanj 2002]. Equations were numerically solved using the 'ode15s' stiff solver within Matlab 6.0 (R13) until concentration levels stopped changing within a tolerance of $10^{-9}$ for at least 5000 seconds. 
Table S1. BER Model Parameters ${ }^{1}$

\begin{tabular}{|c|c|c|c|c|c|c|}
\hline Reaction & \multicolumn{2}{|c|}{$\mathbf{k}_{\text {cat }}\left(\mathrm{s}^{-1}\right)$} & & \multicolumn{3}{|l|}{$K_{M}(n M)$} \\
\hline Ogg1 8-oxoG excision & \multicolumn{2}{|c|}{$1.39 \square 10^{-3}$} & & \multicolumn{3}{|l|}{1836} \\
\hline Ogg1 glycosylase (\& Ape1) & \multicolumn{2}{|c|}{$7.55 \square 10^{-3}$} & $k_{1}$ & \multicolumn{2}{|l|}{8.9} & $K_{1}$ \\
\hline Ogg1 AP lyase & \multicolumn{2}{|c|}{$8.9 \square 10^{-4}$} & & \multicolumn{2}{|l|}{13.7} & \\
\hline Ogg1 AP lyase (\& Ape1) & \multicolumn{2}{|c|}{$1.335 \square 10^{-4}$} & $k_{8}$ & \multicolumn{2}{|l|}{13.7} & \\
\hline Ape1 AP endonuclease & \multicolumn{2}{|c|}{$0.362-10(3.08)^{2}$} & $k_{2}$ & \multicolumn{2}{|c|}{$9.2-230(32.5)$} & $K_{2}$ \\
\hline Ape1 3'-phosphodiesterase & \multicolumn{2}{|l|}{0.05} & $k_{9}$ & \multicolumn{2}{|l|}{130} & $K_{9}$ \\
\hline Pol $\square$ gap-filling & \multicolumn{2}{|c|}{$0.45-0.8(0.625)$} & $k_{3}$ & \multicolumn{2}{|l|}{300} & $K_{3}$ \\
\hline Pol $\square$ dRp lyase & \multicolumn{2}{|l|}{0.075} & $k_{4}$ & \multicolumn{2}{|l|}{500} & $K_{4}$ \\
\hline Lig1/3 ligation $^{3}$ & \multicolumn{2}{|l|}{0.04} & $k_{5}$ & \multicolumn{2}{|l|}{100} & $K_{5}$ \\
\hline Pol $\square$ gap-filling (\& PCNA) & \multicolumn{2}{|l|}{0.0022} & $k_{6}$ & \multicolumn{2}{|l|}{67} & $K_{6}$ \\
\hline Fen1 5'-flap endonuclease & \multicolumn{2}{|l|}{0.39} & & \multicolumn{2}{|l|}{39} & \\
\hline Fen1 5'-endo (\& Ape1) & \multicolumn{2}{|l|}{0.78} & $k_{7}$ & \multicolumn{2}{|l|}{39} & $K_{7}$ \\
\hline \multicolumn{3}{|l|}{ Enzyme } & \multicolumn{2}{|c|}{$\begin{array}{l}\text { Abundance } \\
\text { (molecules/cell) }\end{array}$} & \multicolumn{2}{|c|}{ Conc. (nM) } \\
\hline \multicolumn{2}{|c|}{ 8-oxoguanine DNA glycosylase (Ogg1) } & $e_{1}$ & \multicolumn{2}{|c|}{123,000} & \multicolumn{2}{|c|}{48.8} \\
\hline \multicolumn{2}{|l|}{ AP endonuclease (Ape1) } & $e_{2}$ & \multicolumn{2}{|c|}{$250-350,000(300,000)$} & \multicolumn{2}{|l|}{119} \\
\hline Polymerase $\square($ Polß) & & $e_{3}$ & 50,0 & & 19.8 & \\
\hline Polymerase $\square$ (Pol $\square)$ & & $e_{4}$ & 50,0 & & 19.8 & \\
\hline Flap endonuclase (Fen1) & & $e_{5}$ & 50,0 & & 19.8 & \\
\hline DNA ligase 1 (Lig1) & & $e_{6}$ & 5,00 & & 127 & \\
\hline DNA ligase 3 (Lig3) & & $e_{7}$ & $2-10$ & $(5,000)^{8}$ & 127 & \\
\hline
\end{tabular}

${ }^{1}$ Values from [Sokhansanj 2002], except as noted in text.

${ }^{2}$ Average of values found in literature (in paren.) is used.

3 Lig3 kinetics are assumed equal to Lig1 kinetics.

${ }^{4}$ From [Horton 1995]

${ }^{5}$ Not experimentally derived, assumed equal to Pol $\square$.

${ }^{6}$ Abundance based on quoted range from 2,000 to 10,000 in [Tomkinson 1990] and (Tomkinson, personal communication)

${ }_{8}^{7}$ Assumed to be concentrated within the nucleus.

${ }^{8}$ Assumed equal to Lig1. 
Table S2. Steady State Concentration of Repair Intermediates (Sites/Cell)

\begin{tabular}{ccccc}
\hline $\begin{array}{c}\text { 8-oxoG Formation Rates } \\
\text { (sites/cell/day) }\end{array}$ & $\mathbf{5 0 0}$ & $\mathbf{2 , 0 0 0}$ & $\mathbf{5 , 0 0 0}$ & $\mathbf{1 0 , 0 0 0}$ \\
\hline $\begin{array}{c}\text { 8-oxoG sites } \\
\text { abasic sites }\end{array}$ & 1.4 & 5.6 & 14.0 & 28.2 \\
\hline Pathway B Intermediates & & 13.3 & 16.6 & 22.3 \\
5'dRp-flap, gapped & 38.9 & 44.5 & 55.7 & 74.5 \\
5'dRp-flap, nicked & 0.1 & 0.1 & 0.1 & 0.1 \\
gapped & 0.4 & 0.4 & 0.5 & 0.7 \\
nicked & 0.06 & 0.06 & 0.08 & 0.1 \\
\hline Pathway C Intermediates & & & & \\
flapped (2-7 nt) & 0.06 & 0.07 & 0.09 & 0.1 \\
nicked (2-7 nt) & 0.2 & 0.3 & 0.4 & 0.5 \\
\hline Pathway A Intermediates & & & & \\
3'dRp-flap, gapped & 0.001 & 0.006 & 0.01 & 0.03 \\
gapped & 0.003 & 0.01 & 0.03 & 0.06 \\
nicked & 0.0006 & 0.003 & 0.007 & 0.01 \\
\hline \hline Total Number of Lesions & 52.7 & 64.3 & 87.6 & 126.6 \\
\hline & & & &
\end{tabular}




\section{REFERENCES IN SUPPLEMENTARY MATERIAL}

I. Dianova, V. A. Bohr, L. Gianov, Interaction of human AP endonuclease 1 with flap endonuclease 1 and proliferating cell nuclear antigen involved in long-patch base excision repair, Biochemistry, 40 (2001) 1263912644.

A. R. Evans, M. Limp-Foster, M. R. Kelley, Going APE over ref-1, Mutat. Res., 461 (2000) 83-108.

E. L. Y. Ho and M.S. Satho, Repair of single-strand DNA interruptions by redundant pathways and its implication in cellular sensitivity to DNA-damaging agents, Nucleic Acids Res., 31 (2003) 7032-7040.

J. K. Horton, D. K. Srivastava, B. Z. Zmudzka, S. H. Wilson, Strategic down-regulation of DNA polymerase beta by antisense RNA sensitizes mammalian cells to specific DNA damaging agents, Nucleic Acids. Res., 23 (1995) 3810-3815.

Y. Nakabeppu, Regulation of intracellular localization of human MTH1, OGG1, and MYH proteins for repair of oxidative DNA damage, Prog. Nucleic Acid Res. Mol. Biol., 68 (2001) 75-94.

R. D. Phair and T. Misteli, High mobility of proteins in the mammalian cell nucleus, Nature, 404 (2000) 604609.

T. Saitoh, K. Shinmura, S. Yamaguchi, M. Tani, S. Seki, H. Murakami, Y. Nojima, J. Yokota, Enhancement of OGG1 protein AP lyase activity by increase of APEX protein, Mutat. Res., 486 (2000) 31-40.

B. A. Sokhansanj, G. H. Rodrigue, J. P. Fitch, D.M. Wilson, III, Nucleic Acids Res., 30 (2002) 1817-1825.

A. E. Tomkinson, D. D. Lasko, G. Daly, T. Lindahl, Mammalian DNA ligases, Catalytic domain and size of DNA ligase I, J. Biol. Chem., 265 (1990) 12611-12617. 